\title{
Safety and efficacy of non-steroidal anti-inflammatory drugs to reduce ileus after colorectal surgery
}

\author{
EuroSurg Collaborative*
}

Correspondence to: Mr S. J. Chapman, Room 7.16 Clinical Sciences Building, Leeds Institute of Medical Research at St James's, University of Leeds, Leeds LS9 7TF, UK (e-mail: stephen.chapman@doctors.org.uk; 1 @SJ_Chapman, @EuroSurg)

Background: Ileus is common after elective colorectal surgery, and is associated with increased adverse events and prolonged hospital stay. The aim was to assess the role of non-steroidal anti-inflammatory drugs (NSAIDs) for reducing ileus after surgery.

Methods: A prospective multicentre cohort study was delivered by an international, student- and trainee-led collaborative group. Adult patients undergoing elective colorectal resection between January and April 2018 were included. The primary outcome was time to gastrointestinal recovery, measured using a composite measure of bowel function and tolerance to oral intake. The impact of NSAIDs was explored using Cox regression analyses, including the results of a centre-specific survey of compliance to enhanced recovery principles. Secondary safety outcomes included anastomotic leak rate and acute kidney injury.

Results: A total of 4164 patients were included, with a median age of 68 (i.q.r. 57-75) years (54.9 per cent men). Some 1153 (27.7 per cent) received NSAIDs on postoperative days 1-3, of whom 1061 (92.0 per cent) received non-selective cyclo-oxygenase inhibitors. After adjustment for baseline differences, the mean time to gastrointestinal recovery did not differ significantly between patients who received NSAIDs and those who did not (4.6 versus 4.8 days; hazard ratio $1.04,95$ per cent c.i. 0.96 to $1 \cdot 12 ; P=0.360)$. There were no significant differences in anastomotic leak rate (5.4 versus 4.6 per cent; $P=0.349)$ or acute kidney injury (14.3 versus 13.8 per cent; $P=0.666)$ between the groups. Significantly fewer patients receiving NSAIDs required strong opioid analgesia (35.3 versus 56.7 per cent; $P<0.001)$.

Conclusion: NSAIDs did not reduce the time for gastrointestinal recovery after colorectal surgery, but they were safe and associated with reduced postoperative opioid requirement.

* Members of the EuroSurg Collaborative are co-authors of this study and are listed in Appendix S1
(supporting information)
VIDEO
Www.bjs.co.uk

\section{Introduction}

Ileus is common after colorectal surgery. It occurs in 10-20 per cent of patients after elective colonic resection, making it the most common complication ahead of anastomotic leak and surgical-site infection ${ }^{1}$. In 2014, the Association of Coloproctology in Great Britain and Ireland ${ }^{2}$ identified ileus as an unmet clinical challenge. Its clinical manifestations are profound, contributing to postoperative pain, vomiting and malnutrition. Its burden on healthcare systems is also substantial, leading to a 70 per cent increase in healthcare costs ${ }^{3}$.

Many strategies to reduce ileus have been tested in the past 20 years, but few have resulted in meaningful clinical benefits ${ }^{4}$. This may be attributed to an incomplete understanding of the pathophysiology, and differences between experimental animal models and humans ${ }^{5}$. Current evidence describes a complex relationship between inflammatory, neurogenic and vagal mechanisms, which are exacerbated by the effects of opioid analgesia and other homeostatic imbalances ${ }^{6}$. To date, the most promising strategies have been those that aim to rationalize opioid-based analgesia (such as $\mu$-receptor antagonists), and to moderate the postoperative inflammatory response (such as enhanced recovery protocols) ${ }^{4}$.

Non-steroidal anti-inflammatory drugs (NSAIDs) are recommended in enhanced recovery protocols for their opioid-sparing and anti-inflammatory properties ${ }^{7}$. These 
may have benefits for the recovery of gastrointestinal function. Recent meta-analyses ${ }^{8,9}$ of RCTs demonstrated a significant pooled benefit of NSAIDs in reducing ileus after colorectal surgery. However, the use of NSAIDs in this setting is controversial owing to the risk of acute kidney injury and conflicting reports of increased anastomotic leak rates ${ }^{10,11}$. NSAIDs may represent a simple and cost-effective intervention to reduce ileus.

The primary aim of this study was to explore the relationship between NSAIDs and the recovery of gastrointestinal function (resolution of ileus) after colorectal surgery. It also aimed to examine key safety outcomes, when NSAIDs are administered in the early postoperative phase.

\section{Methods}

The study protocol was developed by an international management group, with input from patient representatives. The protocol was registered prospectively at the Research Registry Service (https://www.researchregistry.com) (UIN 3072) and published before the study started ${ }^{12}$. Study approvals and requirements for individual patient consent were satisfied according to country-specific regulations. This manuscript is the first analysis of the international IMAGINE study and is reported in line with the STROBE statement ${ }^{13}$.

\section{Study design}

A prospective multicentre observational study was delivered by a student- and trainee-led collaborative group with a track record of international research ${ }^{14}$. The study design was informed by an external pilot study in a comparable patient cohort in the $\mathrm{UK}^{15}$. A 17-item, prestudy survey of compliance to enhanced recovery principles was completed by representatives of participating hospitals before the start of data collection (Table S1, supporting information) and used to produce a score (enhanced recovery after surgery (ERAS) score). To determine the accuracy and completeness of data, an independent validation exercise was preplanned in at least 10 per cent of centres. This comprised two domains; the first was data accuracy, determined by assessing the accuracy of ten planned data points; the second was case ascertainment, determined by assessing the accuracy of participant eligibility. No change to the final data set was planned based on this exercise, but an accuracy of at least 95 per cent in each domain was considered sufficient for validation.

\section{Study setting}

Hospitals performing elective colorectal surgery in Europe, Australasia and South Africa were eligible to enrol in the study. Each hospital enrolled consecutive patients in up to three 14-day data collection intervals between January and April 2018.

\section{Eligibility criteria}

Adult patients having elective colorectal resection via open, laparoscopic or robotic approaches for any indication (malignant or benign) were eligible. Procedures performed via a transanal approach, and those for primary gynaecological, hepatobiliary, urological or vascular pathologies were excluded. Return to theatre was considered to represent an acute episode and was not eligible. Elective appendicectomy was excluded, unless a more extensive colorectal resection was undertaken. Patients undergoing restoration of intestinal continuity were included, but will be reported elsewhere ${ }^{12}$.

\section{Outcome measures}

The primary outcome was time to gastrointestinal recovery. The validated GI-2 composite measure was used, defined as the time taken for patients to tolerate solid food and to pass stool ${ }^{16}$. This included soft food, but not liquids. Secondary outcomes were postoperative complications occurring within 30 days of surgery. The Clavien-Dindo system ${ }^{17}$ was used to classify each patient's most severe encountered complication: no complication (grade 0), minor complication (grades I-II) or major complication (grades III-V). Anastomotic leak was defined as bowel leakage detected radiologically, or at the time of reoperation. Acute kidney injury was defined according to the Kidney Disease Improving Global Outcomes serum creatinine-based criteria ${ }^{18}$.

\section{Explanatory variables}

The main variable of interest was early administration of NSAIDs after surgery. Patients were considered to have received a course of NSAIDs if received on at least 2 days (at least 1 dose per day) within the first 3 days after operation. Variables including ASA fitness grade, BMI and smoking status were collected for risk adjustment. Other variables of interest were those known to affect gastrointestinal recovery after surgery: cardiovascular and metabolic diseases (chronic obstructive pulmonary disease, ischaemic heart disease, peripheral artery disease and diabetes mellitus), previous abdominal surgery, operative approach, transfusion of red cells, postoperative administration of strong opioids (defined as at least 2 days of oral or parenteral treatment within the first 3 days after surgery) 
and centre-specific compliance with enhanced recovery principles ${ }^{19}$. Examples of strong and weak opioids relevant to this study are provided in Table S2 (supporting information).

\section{Statistical analysis}

A detailed statistical analysis plan and power calculation has been published previously ${ }^{12}$. The study was powered based on a comparison of GI-2 between patients who received NSAIDs and those who did not. Based on data from an external pilot study, it was estimated that it would be feasible to recruit approximately 3500 participants during the study interval, and that the NSAID administration rate would be approximately 15 per cent ${ }^{20}$. A power calculation assuming these values returned a minimal detectable difference in GI-2 of 0.2 days between groups ( 5 per cent $\alpha ; 80$ per cent power). A difference in GI-2 of 1 day was considered clinically meaningful when applied to real practice, so the study was adequately powered for this clinical difference.

Patient demographics, perioperative variables and safety outcomes were compared between treatment groups using the $\chi^{2}$ test for nominal variables, and Mann-Whitney $U$ test for ordinal or continuous variables.

A Cox regression model was used for univariable analysis, with the differences across treatment groups quantified using Kaplan-Meier curves and mean times to GI-2. Multivariable Cox regression models were then produced to identify independent predictors of the time to GI-2. The maximum follow-up was 10 days; patients were censored at this point if GI-2 had not been achieved. As achieving GI-2 was treated as the event in the analyses, a hazard ratio (HR) greater than 1.00 was indicative of a greater hazard of achieving GI-2 and, accordingly, a shorter time to GI-2.

In the main analysis, patients were censored at discharge or death in hospital, as the time to GI-2 was not known for these patients. However, this may have violated the assumption of non-informative censoring. Patients who were discharged would have been deemed fit at this point, and would probably have achieved GI-2 soon after. In the case of inpatient death, the patient would have had no opportunity to achieve GI-2, even if follow-up had been extended indefinitely. As such, a sensitivity analysis was undertaken that treated patients discharged before achieving GI-2 as having achieved GI-2 on the day of discharge. Those who died in hospital before achieving GI-2 were treated as not having achieved GI-2 by day 10, rather than being censored at the point of death in this sensitivity analysis.
A multivariable analysis of safety outcomes was carried out to assess whether any of these were associated independently with NSAID use. Duration of hospital stay was highly skewed, so was dichotomized for analysis, based on the median value. All outcomes were analysed using multivariable binary logistic regression models. The NSAID group was entered into each model as a predictor, with a backwards stepwise approach used to select other preoperative and perioperative factors that were associated independently with the outcome being considered.

$P<0.050$ was deemed to be indicative of statistical significance throughout. All analyses were performed using SPSS ${ }^{\circledR}$ version 22 (IBM, Armonk, New York, USA).

\section{Results}

Data were available for 4164 patients, with a median age of 68 (i.q.r. 57-75) years, of whom 54.9 per cent were men. An anastomosis was constructed in 3638 patients $(87.4$ per cent). Between postoperative days 1 and 3, 1153 patients (27.7 per cent) received an early course of NSAIDs: non-selective cyclo-oxygenase (COX) inhibitors in 1061 patients (92.0 per cent) and COX-2-selective agents in 92 (8.0 per cent). Data validation was performed using information on 892 patients from 111 centres $(21.4$ per cent sample of the total cohort), with 95.9 per cent data accuracy and 99.7 per cent case ascertainment.

\section{Baseline data}

Some differences in baseline patient variables were observed between treatment groups (Table 1). Patients receiving NSAIDs were significantly younger (median 66 versus 68 years; $P<0 \cdot 001)$, more likely to be smokers (17.9 versus 15.2 per cent; $P=0.033)$ and had lower ASA grades $(P=0.040)$ and ERAS scores $(P<0.001)$ than those not treated with NSAIDs. A significant difference in surgical pathology was also identified $(P=0.013)$; NSAIDs were used more frequently among patients treated for malignancy.

\section{Operative and postoperative treatment}

There were also differences in operative and postoperative factors between treatment groups (Table 2). Patients receiving NSAIDs were more likely to have had open surgery $(40.3$ versus 35.6 per cent; $P=0.001)$ and to have undergone a left colonic resection (25.0 versus 21.7 per cent; $P=0 \cdot 024)$. Where C-reactive protein (CRP) levels were recorded between postoperative days 1 and 3 , these were significantly lower in patients treated with NSAIDs (median 100 versus $118 \mathrm{mg} / \mathrm{l} ; P<0.001$ ). Patients receiving 


\begin{tabular}{|c|c|c|c|c|}
\hline & \multirow[b]{2}{*}{ No. of patients } & \multicolumn{2}{|c|}{ NSAID use } & \multirow[b]{2}{*}{$\boldsymbol{P} \dagger$} \\
\hline & & No & Yes & \\
\hline Age (years)* & 4162 & $68(57-75)$ & $66(55-74)$ & $<0.001 \ddagger$ \\
\hline Men & 4164 & 1654 (54.9) & $634(55.0)$ & 1.000 \\
\hline BMI $\left(\mathrm{kg} / \mathrm{m}^{2}\right)$ & 4159 & & & $0.050 \div \S$ \\
\hline$<18.5$ & & $79(2 \cdot 6)$ & $37(3 \cdot 2)$ & \\
\hline $18 \cdot 5-24.9$ & & $1168(38 \cdot 8)$ & $476(41.4)$ & \\
\hline $25 \cdot 0-30 \cdot 0$ & & $1135(37 \cdot 7)$ & $419(36 \cdot 4)$ & \\
\hline$>30.0$ & & $626(20.8)$ & $219(19.0)$ & \\
\hline Current smoker & 4155 & $455(15 \cdot 2)$ & $206(17.9)$ & 0.033 \\
\hline ASA fitness grade & 4160 & & & $0.040 \div \S$ \\
\hline I & & $320(10 \cdot 6)$ & $147(12 \cdot 8)$ & \\
\hline II & & $1700(56 \cdot 5)$ & $656(56 \cdot 9)$ & \\
\hline III & & $904(30 \cdot 1)$ & $318(27 \cdot 6)$ & \\
\hline IV-V & & $84(2 \cdot 8)$ & $31(2 \cdot 7)$ & \\
\hline Previous abdominal surgery & 4163 & $1206(40 \cdot 1)$ & $497(43 \cdot 1)$ & 0.078 \\
\hline Existing stoma & 4163 & & & 0.351 \\
\hline No & & $2784(92.5)$ & $1079(93.6)$ & \\
\hline Ileostomy & & $116(3.9)$ & $42(3 \cdot 6)$ & \\
\hline Colostomy & & $110(3 \cdot 7)$ & $32(2 \cdot 8)$ & \\
\hline History of IHD & 4164 & $361(12 \cdot 0)$ & $131(11.4)$ & 0.592 \\
\hline History of PAD & 4164 & $177(5 \cdot 9)$ & $82(7 \cdot 1)$ & $0 \cdot 151$ \\
\hline History of COPD & 4164 & $214(7 \cdot 1)$ & $76(6 \cdot 6)$ & 0.587 \\
\hline History of diabetes & 4164 & & & 0.082 \\
\hline No & & $2528(84 \cdot 0)$ & $960(83.3)$ & \\
\hline Diet/tablet-controlled & & $387(12 \cdot 9)$ & $140(12 \cdot 1)$ & \\
\hline Insulin-controlled & & $96(3.2)$ & $53(4 \cdot 6)$ & \\
\hline ERAS score & 4164 & & & $<0.001 \ddagger \S$ \\
\hline$<12$ & & $564(18 \cdot 7)$ & $367(31 \cdot 8)$ & \\
\hline $12-13$ & & $718(23 \cdot 8)$ & $274(23 \cdot 8)$ & \\
\hline $14-15$ & & $725(24 \cdot 1)$ & $275(23 \cdot 9)$ & \\
\hline$\geq 16$ & & $1004(33 \cdot 3)$ & $237(20 \cdot 6)$ & \\
\hline Underlying pathology & 4164 & & & 0.013 \\
\hline Diverticular disease & & $160(5 \cdot 3)$ & $79(6.9)$ & \\
\hline Inflammatory bowel disease & & $309(10 \cdot 3)$ & $91(7.9)$ & \\
\hline Malignancy & & $2354(78 \cdot 2)$ & $925(80 \cdot 2)$ & \\
\hline Other benign disease & & $188(6 \cdot 2)$ & $58(5 \cdot 0)$ & \\
\hline
\end{tabular}

Values in parentheses are percentages unless indicated otherwise; *values are median (i.q.r.). Patients were considered to have received a course of non-steroidal anti-inflammatory drugs (NSAIDs) if received on at least 2 of the first 3 days after surgery. IHD, ischaemic heart disease; PAD, peripheral artery disease; COPD, chronic obstructive pulmonary disease; ERAS, enhanced recovery after surgery. $\dagger \chi^{2}$ test, except $\ddagger$ Mann-Whitney $U$ test ( $\S$ variable is ordinal).

NSAIDs were significantly less likely to be treated with strong opioids ( 35.3 versus 56.7 per cent; $P<0.001)$, intravenous patient-controlled analgesia $(25.0$ versus $41 \cdot 1$ per cent; $P<0.001)$, and wound catheters $(3.3$ versus 5.9 per cent; $P<0.001)$. However, patients in the NSAID group were significantly more likely to receive prokinetic drugs ( 40.8 versus 31.0 per cent; $P<0.001$ ) and to require red cell transfusion $(10.8$ versus 8.6 per cent; $P=0.036)$ after operation.

\section{Gastrointestinal recovery}

A total of 3716 patients ( 89.2 per cent) achieved GI-2 within the first 10 days after surgery. Of the remainder, 189 ( 4.5 per cent) achieved GI- 2 after more than 10 days, 232 (5.6 per cent) were discharged before day 10 without having achieved GI-2, and 27 (0.6 per cent) died as inpatients before achieving the outcome. Univariable analyses of the times to GI-2 are shown in Fig. 1. In the 


\begin{tabular}{|c|c|c|c|c|}
\hline & \multirow[b]{2}{*}{ No. of patients } & \multicolumn{2}{|c|}{ NSAID use } & \multirow[b]{2}{*}{$P \ddagger$} \\
\hline & & No & Yes & \\
\hline \multicolumn{5}{|l|}{ Operative factors } \\
\hline Operative approach & 4164 & & & 0.001 \\
\hline Minimally invasive & & $1685(56 \cdot 0)$ & $621(53 \cdot 9)$ & \\
\hline Minimally invasive converted to open & & $254(8.4)$ & $67(5 \cdot 8)$ & \\
\hline Open & & $1072(35 \cdot 6)$ & $465(40 \cdot 3)$ & \\
\hline Resection type & 4157 & & & 0.024 \\
\hline Colonic - right & & $1109(36 \cdot 9)$ & $400(34 \cdot 8)$ & \\
\hline Colonic - left & & $653(21 \cdot 7)$ & $287(25 \cdot 0)$ & \\
\hline Rectal & & $1047(34 \cdot 8)$ & $408(35.5)$ & \\
\hline Total colectomy $\dagger$ & & $198(6 \cdot 6)$ & $55(4 \cdot 8)$ & \\
\hline Formation of new stoma & 4164 & & & 0.104 \\
\hline No & & $2032(67.5)$ & $817(70.9)$ & \\
\hline lleostomy & & $551(18 \cdot 3)$ & $193(16 \cdot 7)$ & \\
\hline Colostomy & & $428(14 \cdot 2)$ & $143(12 \cdot 4)$ & \\
\hline \multicolumn{5}{|l|}{ CRP measurement } \\
\hline CRP recorded (POD 1-3) & 4161 & $2378(79 \cdot 0)$ & $785(68 \cdot 2)$ & $<0.001$ \\
\hline CRP level on POD 1-3 (mg/l) & 3163 & $118(67-191)$ & $100(37-169)$ & $<0.001 \S$ \\
\hline \multicolumn{5}{|l|}{ Treatment on POD 1-10 } \\
\hline Strong opioid for $>1$ day on POD $1-3$ & 4164 & $1707(56 \cdot 7)$ & $407(35 \cdot 3)$ & $<0.001$ \\
\hline Epidural catheter & 4163 & $561(18 \cdot 6)$ & $201(17.4)$ & 0.395 \\
\hline Intravenous patient-controlled analgesia & 4163 & $1237(41 \cdot 1)$ & $288(25 \cdot 0)$ & $<0.001$ \\
\hline Wound catheter & 4163 & $179(5 \cdot 9)$ & $38(3 \cdot 3)$ & $<0.001$ \\
\hline Chewing gum & 4160 & $152(5 \cdot 1)$ & $71(6 \cdot 2)$ & 0.166 \\
\hline Prokinetic drugs & 4162 & $932(31 \cdot 0)$ & $471(40 \cdot 8)$ & $<0.001$ \\
\hline Red blood cells & 4164 & $260(8 \cdot 6)$ & $124(10 \cdot 8)$ & 0.036 \\
\hline$\mu$-Opioid antagonists & 4163 & $41(1 \cdot 4)$ & $23(2 \cdot 0)$ & 0.158 \\
\hline
\end{tabular}

Values in parentheses are percentages unless indicated otherwise; *values are median (i.q.r.). Patients were considered to have received a course of non-steroidal anti-inflammatory drugs (NSAID) if received on at least 2 of the first 3 days after surgery. $\dagger$ Includes total and subtotal colonic resection. CRP, C-reactive protein; POD, postoperative day. $\ddagger \chi^{2}$ test, except $\$$ Mann-Whitney $U$ test.

main analysis, the time to GI-2 was significantly shorter in patients treated with NSAIDs, with a mean of 4.6 days compared with 4.8 days in those not receiving NSAIDs (HR $1 \cdot 08,95$ per cent c.i. $1 \cdot 00$ to $1 \cdot 16 ; P=0 \cdot 043$ ). Sensitivity analysis returned a similar difference, but this did not reach significance (mean 4.5 versus 4.7 days; HR 1.06, 0.99 to $1.14 ; P=0.088)$.

\section{Multivariable analysis}

Because a range of demographic and treatment factors differed between the treatment groups, a multivariable analysis was undertaken to assess whether NSAID use was independently associated with GI-2 (Tables $S 3$ and $S 4$, supporting information). The main analysis showed female sex $(P=0.009)$, insulin-controlled diabetes $(P=0.009)$, increasing ERAS scores $(P=0.003)$, minimally invasive operative approach $(P<0.001)$ and the formation of a new ileostomy $(P<0 \cdot 001)$ to be associated with a significantly shorter time to GI-2. Longer times to GI-2 were observed in patients with a higher CRP level on postoperative days $1-3(P<0.001)$, and in those treated with strong opioids $(P<0.001)$, epidural catheters $(P=0.036)$, intravenous patient-controlled analgesia $(P=0.019)$, prokinetic drugs $(P=0.009)$ and red cell transfusion on postoperative days $1-10(P=0.005)$. After accounting for these factors, the use of NSAIDs was not independently associated with the time to GI-2 (HR 1.04, 95 per cent c.i. 0.96 to 1.12 ; $P=0.360)$. The sensitivity analysis yielded consistent results (HR 1.04, 0.96 to $1.11 ; P=0.353$ ).

\section{Safety outcomes}

On univariable analysis, no significant difference in duration of hospital stay was observed between patients who were treated with NSAIDs and those who were not: median 8 (i.q.r. $6-11)$ days in both groups $(P=0 \cdot 635)$ 


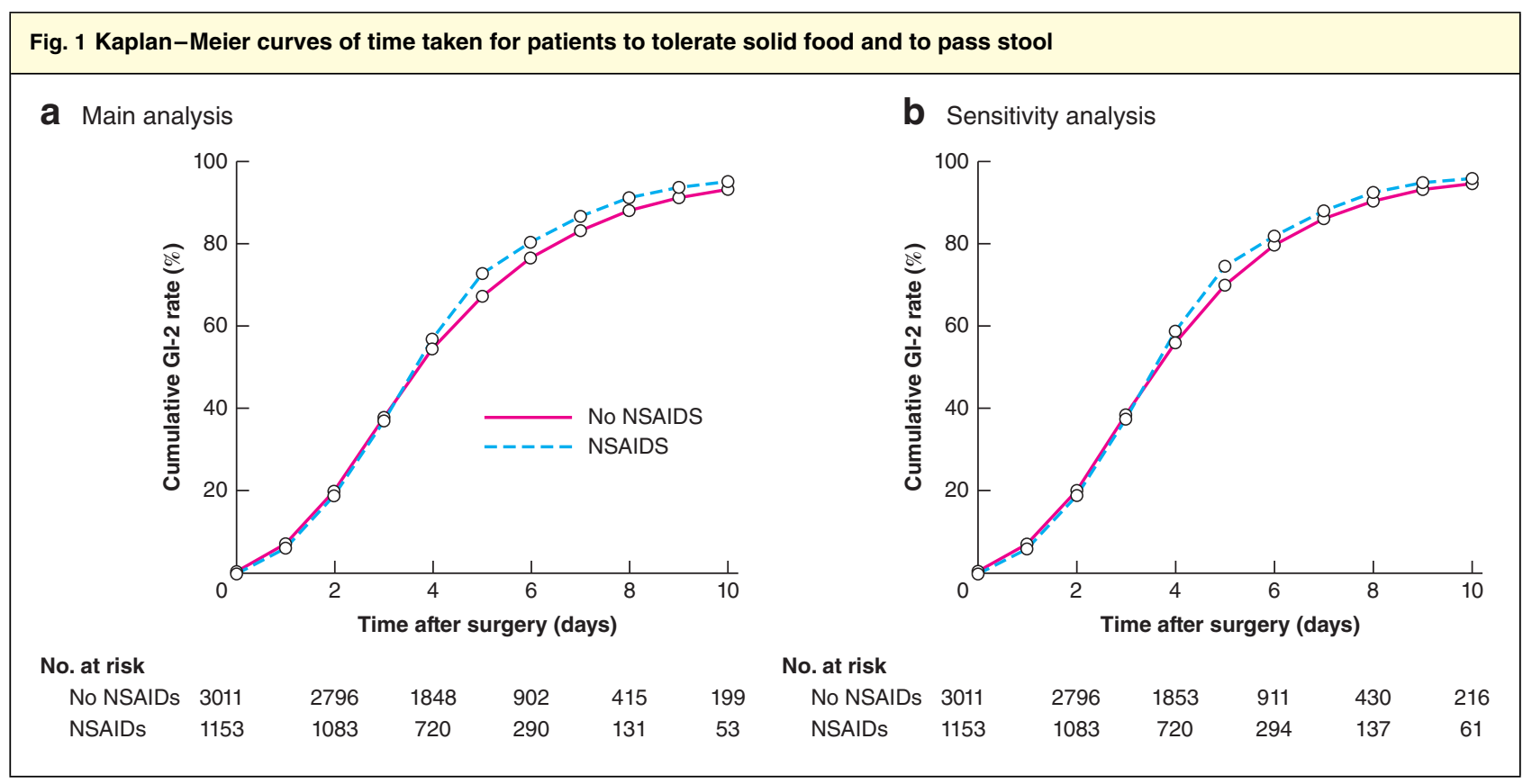

Kaplan-Meier estimated cumulative rates of patients being able to tolerate solid food and to pass stool (GI-2 composite outcome) on each postoperative day: a main analysis with patients censored at discharge or death without achieving GI-2, and $\mathbf{b}$ sensitivity analysis in which patients discharged without GI-2 were treated as achieving the outcome on the day of discharge, with inpatient deaths treated as being censored on postoperative day 11 . The points have been connected using straight lines, rather than the conventional stepped lines to highlight the differences between groups more clearly. Patients were considered to have received a course of non-steroidal anti-inflammatory drugs (NSAIDs) if received on at least 2 of the first 3 days after surgery. a $P=0 \cdot 043$, b $P=0.088$ (univariable Cox regression analysis).

\begin{tabular}{|c|c|c|c|c|}
\hline & \multirow[b]{2}{*}{ No. of patients } & \multicolumn{2}{|c|}{ NSAID use } & \multirow[b]{2}{*}{$\boldsymbol{P} \S$} \\
\hline & & No & Yes & \\
\hline Postoperative duration of hospital stay (days) ${ }^{\star} \dagger$ & 4156 & $8(6-11)$ & $8(6-11)$ & $0.635 \rrbracket$ \\
\hline Anastomotic leak $\ddagger$ & 3628 & $120(4 \cdot 6)$ & $56(5 \cdot 4)$ & 0.349 \\
\hline Intra-abdominal collection & 4161 & $194(6 \cdot 4)$ & $66(5 \cdot 7)$ & 0.431 \\
\hline Pneumonia & 4161 & $146(4.9)$ & $39(3.4)$ & 0.043 \\
\hline Acute kidney injury & 3710 & $377(13 \cdot 8)$ & $139(14 \cdot 3)$ & 0.666 \\
\hline Readmission & 4151 & $265(8 \cdot 8)$ & $109(9.5)$ & 0.506 \\
\hline Complications (Clavien-Dindo grade) & 4160 & & & $0.251 \Phi$ \\
\hline 0 & & $1370(45 \cdot 6)$ & $540(46 \cdot 8)$ & \\
\hline I-II & & $1314(43 \cdot 7)$ & $509(44 \cdot 1)$ & \\
\hline III-V & & $323(10 \cdot 7)$ & $104(9 \cdot 0)$ & \\
\hline
\end{tabular}

Values in parentheses are percentages unless indicated otherwise; *values are median (i.q.r.). $†$ Values truncated at 30 days. $\ddagger$ Excluding 526 patients with no anastomosis. $\S \chi^{2}$ test, except I[Mann-Whitney $U$ test (variable is ordinal).

(Table 3). The rate of anastomotic leak was similar in the NSAID and no-NSAID groups ( 5.4 versus 4.6 per cent respectively; $P=0.349)$; in subgroup analyses, this difference remained non-significant for each type of resection (Table S5, supporting information). The rates of intra-abdominal collection (5.7 versus 6.4 per cent; $P=0.431)$, acute kidney injury (14.3 versus 13.8 per cent $P=0.666)$ and readmission $(9.5$ versus 8.8 per cent;
$P=0.506)$ were similar in both groups, although patients treated with NSAIDs had a significantly lower rate of pneumonia ( 3.4 versus 4.9 per cent; $P=0.043)$. There was no significant difference in overall rates of complications $(P=0.251)$; minor complications occurred in 44.1 versus 43.7 per cent, and major complications in 9.0 versus 10.7 per cent, of patients receiving NSAIDs and no NSAIDs respectively. 


\begin{tabular}{|c|c|c|c|c|}
\hline & \multicolumn{2}{|c|}{ Univariable analysis } & \multicolumn{2}{|c|}{ Multivariable analysis } \\
\hline & Odds ratio & $\boldsymbol{P}$ & Odds ratio & $P$ \\
\hline Postoperative hospital stay $>8$ days & $0.93(0.81,1.06)$ & 0.279 & $0.87(0.75,1.02)$ & 0.083 \\
\hline Anastomotic leak* & $1.17(0.84,1.61)$ & 0.357 & $1.14(0.82,1.59)$ & 0.424 \\
\hline Intra-abdominal collection & $0.88(0.66,1.18)$ & 0.387 & $0.87(0.65,1.16)$ & 0.338 \\
\hline Pneumonia & $0.69(0.48,0.98)$ & 0.040 & $0.72(0.50,1.04)$ & 0.077 \\
\hline Acute kidney injury & $1.05(0.85,1.30)$ & 0.648 & $1.04(0.83,1.29)$ & 0.755 \\
\hline Readmission & $1.08(0.86,1.37)$ & 0.507 & $1.09(0.86,1.38)$ & 0.480 \\
\hline Clavien-Dindo grade III-V complications & $0.82(0.65,1.04)$ & $0 \cdot 102$ & $0.83(0.65,1.04)$ & 0.110 \\
\hline
\end{tabular}

Values in parentheses are 95 per cent confidence intervals. Odds ratios are shown for the non-steroidal anti-inflammatory drug (NSAID) group versus the no-NSAID group. *Excluding 526 patients with no anastomosis. The multivariable models used a backwards stepwise approach to variable selection, and considered NSAID use alongside all preoperative and perioperative factors from Tables $S 3$ and $S 4$ (supporting information). Full information on the multivariable models is available in Tables $S 6-S 8$ (supporting information).

The analysis was repeated using a multivariable approach, to account for the potentially confounding effect of other preoperative and perioperative factors. The results were consistent with those of the univariable analyses, with no significant differences in safety outcomes between the treatment groups (Table 4; Tables $S 6-S 8$, supporting information). The difference in rates of pneumonia identified on univariable analysis narrowly missed significance in the multivariable model, with an odds ratio (OR) of 0.72 (95 per cent c.i. 0.50 to $1.04 ; P=0.077$ ) for the NSAID versus no-NSAID group.

\section{Discussion}

This large observational study provided validated data on the safety of NSAIDs after colorectal surgery and their relationship with gastrointestinal recovery. Their early use after surgery was not associated with increased rates of anastomotic leak or acute kidney injury. NSAID use was not associated with significantly improved gastrointestinal recovery in multivariable models, but was associated with a reduced need for strong opioid analgesia after surgery.

The use of NSAIDs after colorectal surgery is controversial. NSAIDs are recommended by enhanced recovery protocols to optimize pain management and to reduce opioid-related adverse effects ${ }^{7}$. They may also improve the recovery of bowel function; a recent meta-analysis ${ }^{8}$ of high-quality RCTs demonstrated that NSAIDs reduce the times to first flatus and stool by 18 and $10 \mathrm{~h}$ respectively. The mechanism for this is unclear, but current evidence suggests a combination of decreased $\mu$-receptor agonism and inhibition of COX enzymes, which reduce neuromuscular dysfunction ${ }^{6}$. In contrast, reservations over the use of NSAIDs are common. NSAIDs may increase the risk of acute kidney injury, which is common after major abdominal surgery (14.2 per cent), and associated with excessive 1-year mortality ${ }^{21,22}$. NSAIDs are also known to inhibit platelet aggregation, and were associated with a small increase in red cell transfusion in the present study $(10.8$ versus 8.6 per cent in NSAID and no-NSAID groups respectively); this was in the absence of excess rates of complications overall. Finally, previous studies ${ }^{10,11}$ have associated NSAIDs with increased anastomotic leak after elective and urgent colorectal resection. In a recent meta-analysis ${ }^{23}$, diclofenac was associated with an increased rate of leak (OR 2.79, 95 per cent c.i. 1.96 to 3.96) but ketorolac was not (OR 1.36, 0.89 to 2.06). This association remains inconclusive and has yet to be corroborated in studies with a low risk of bias.

Fewer patients who received NSAIDs in the present study required strong opioid analgesia. The prescription of opioids after surgery has become a topical issue, owing to their profile of adverse effects and contribution to national opioid epidemics ${ }^{24}$. Many interventions to reduce opioid use after surgery have been explored, including use of epidural and wound catheters. Unfortunately, many of these are invasive, are associated with infective complications, or are restrictive for mobility and enhanced recovery. Despite the apparent association between NSAIDs and reduced opioid requirements, only one-quarter of patients received a relevant dose in the present study, which is consistent with previous reports ${ }^{19}$. This is surprising as NSAIDs are widely recommended by international enhanced recovery guidelines ${ }^{7}$. It is possible that this reflects a lack of equipoise across the colorectal community, influenced by conflicting safety data. Differences between international subcommunities cannot be excluded. Although this study did not consider the role of specific NSAIDs, it adds to a growing body of data that support their safety. 
Strengths of the present study are recognized. First, the definition of ileus is variable and often arbitrary, which is problematic for performing robust clinical trials and commissioning new treatments in clinical practice ${ }^{25}$. A validated measure of gastrointestinal recovery (GI-2) was used here, which reduced variation in defining ileus across a multicentre centre setting ${ }^{16}$. Second, in recognizing that some patients are discharged before achieving GI-2 (before the passage of stool), a prospective sensitivity analysis was preplanned $^{12}$. This helped to increase the generalizability of the study findings across settings, where variable criteria for hospital discharge may exist. Other strengths of the study include its delivery across a student- and trainee-led collaborative network (facilitating a large and real-life snapshot of clinical practice), a prospective validation exercise (confirming the quality of data arising from multiple sources), and the administration of a centre-specific survey of enhanced recovery (allowing clinically relevant adjustment of results within statistical models).

The main weakness of the study was its observational design, with the decision to prescribe NSAIDs being at the discretion of the clinical team. As such, there is the potential for selection bias, highlighted by the difference in patient demographics and postoperative characteristics between treatment groups. To account for this, multivariable analyses were used to adjust for potentially confounding factors. However, there are likely to be other important variables that were not measured or adjusted for in this study, meaning that residual selection bias may persist. Another potential weakness was the statistical power of the analysis. A sample size calculation determined that a sample of 3500 would be sufficient to detect a difference in time to GI- 2 of 0.2 days between groups. This target was satisfied, but the calculation did not account for the presence of selection bias, which, after multivariable adjustment, produced a smaller adjusted effect of NSAIDs. Despite this, the observed difference of 0.2 days in univariable analysis was small. Even if the statistical power were increased, the clinical relevance of such a small effect size would be questionable. Finally, the use of NSAIDs was considered relevant only if they were administered in the early postoperative phase (days $1-3$ ). Although the pathophysiology of anastomotic leak is not necessarily homogeneous in all settings, this is justified as the impact of NSAIDs on normal anastomotic healing (reduced anastomotic COX-2 level) is evident by the third day after operation in preclinical models ${ }^{26}$.

Although the present observational data do not support the efficacy of NSAIDs to reduce ileus after colorectal surgery, there remains a good case for their use in the postoperative setting. Previous data relating to the risk of acute kidney injury are strong and the use of NSAIDs in this setting must be done according to appropriate patient selection. Previous data on the risk of anastomotic leak are inconsistent and not supported by the present findings. For as long as NSAIDs are endorsed by evidence-based enhanced recovery guidelines, their use remains justified. Moving forward, an assessment of equipoise is required to explore factors that may influence the use of NSAIDs after colorectal surgery.

\section{Acknowledgements}

The authors are grateful to the European Society of Coloproctology (ESCP) Executive for providing facilities to host collaborator meetings in Berlin (September 2017) and Nice (September 2018); the ESCP Cohort Studies and Audits Committee for their review of the study protocol; and the Dukes Club (UK), the Portuguese Surgical Research Collaborative, the Young Group of the Italian Society of Colorectal Surgery, the Italian Surgical Research Group, the Australasian Students' Surgical Association, and the Clinical Trials Network of Australia and New Zealand for assistance with study dissemination. They thank the Birmingham Surgical Trials Consortium at the University of Birmingham (UK) for support with online data capture, and the Yorkshire Surgical Research Collaborative (UK) for access to unpublished data.

Disclosure: The authors declare no conflict of interest.

\section{References}

1 Scarborough JE, Schumacher J, Kent KC, Heise CP, Greenberg CC. Associations of specific postoperative complications with outcomes after elective colon resection: a procedure-targeted approach toward surgical quality improvement. JAMA Surg 2017; 152: e164681.

2 Tiernan J, Cook A, Geh I, George B, Magill L, Northover J et al. Use of a modified Delphi approach to develop research priorities for the Association of Coloproctology of Great Britain and Ireland. Colorectal Dis 2014; 16 : 965-970.

3 Mao H, Milne TGE, O'Grady G, Vather R, Edlin R, Bissett I. Prolonged postoperative ileus significantly increases the cost of inpatient stay for patients undergoing elective colorectal surgery: results of a multivariate analysis of prospective data at a single institution. Dis Colon Rectum 2019; 62: 631-637.

4 Chapman SJ, Pericleous A, Downey C, Jayne DG. Postoperative ileus following major colorectal surgery. $\mathrm{Br}$ 7 Surg 2018; 105: 797-810.

5 Chapman SJ, Wells CI. Challenges in ileus research. Colorectal Dis 2018; 20: 639.

6 Boeckxstaens GE, de Jonge WJ. Neuroimmune mechanisms in postoperative ileus. Gut 2009; 58: 1300-1311. 
7 Gustafsson UO, Scott MJ, Hubner M, Nygren J, Demartines N, Francis N et al. Guidelines for perioperative care in elective colorectal surgery: enhanced recovery after surgery (ERAS ${ }^{\circledR}$ ) society recommendations: 2018 . World 7 Surg 2019; 43: 659-695.

8 Chapman SJ, Garner JJ, Drake TM, Aldaffaa M, Jayne DG. Systematic review and meta-analysis of nonsteroidal anti-inflammatory drugs to improve GI recovery after colorectal surgery. Dis Colon Rectum 2019; 62: 248-256.

9 Milne TGE, Jaung R, O'Grady G, Bissett IP. Nonsteroidal anti-inflammatory drugs reduce the time to recovery of gut function after elective colorectal surgery: a systematic review and meta-analysis. Colorectal Dis 2018; 20: O190-O198.

10 Klein M, Gögenur I, Rosenberg J. Postoperative use of non-steroidal anti-inflammatory drugs in patients with anastomotic leakage requiring reoperation after colorectal resection: cohort study based on prospective data. BMF 2012; 345: e6166.

11 Hakkarainen TW, Steele SR, Bastaworous A, Dellinger EP, Farrokhi E, Farjah F et al. Nonsteroidal anti-inflammatory drugs and the risk for anastomotic failure: a report from Washington State's Surgical Care and Outcomes Assessment Program (SCOAP). 7AMA Surg 2015; 150: 223-228.

12 Chapman SJ; EuroSurg Collaborative. Ileus management International (IMAGINE): protocol for a multicentre, observational study of ileus after colorectal surgery. Colorectal Dis 2018; 20: O17-O25.

13 von Elm E, Altman DG, Egger M, Pocock SJ, Gøtzsche PC, Vandenbroucke JP; STROBE Initiative. The strengthening the reporting of observational studies in epidemiology (STROBE) statement: guidelines for reporting observational studies. PLoS Med 2007; 4: e296.

14 EuroSurg Collaborative. EuroSurg: a new European student-driven research network in surgery. Colorectal Dis 2016; 18: 214-215.

15 Yorkshire Surgical Research Collaborative. Multicentre observational study of gastrointestinal recovery after elective colorectal surgery. Colorectal Dis 2018; 20: 536-544.

16 van Bree SH, Bemelman WA, Hollmann MW, Zwinderman AH, Matteoli G, El Temna S et al. Identification of clinical outcome measures for recovery of gastrointestinal motility in postoperative ileus. Ann Surg 2014; 259: 708-714.
17 Dindo D, Demartines N, Clavien PA. Classification of surgical complications: a new proposal with evaluation in a cohort of 6336 patients and results of a survey. Ann Surg 2004; 240: 205-213.

18 Kidney Disease Improving Global Outcomes. KDIGO clinical practice guideline for acute kidney injury. Kidney Int 2012; 2(Suppl 1): 1-138.

19 Wolthuis AM, Bislenghi G, Lambrecht M, Fieuws S, de Buck van Overstraeten A, Boeckxstaens G et al. Preoperative risk factors for prolonged postoperative ileus after colorectal resection. Int 7 Colorectal Dis 2017; 32: 883-890.

20 STARSurg Collaborative. Impact of postoperative non-steroidal anti-inflammatory drugs on adverse events after gastrointestinal surgery. Br 7 Surg 2014; 101: $1413-1423$.

21 O'Connor ME, Hewson RW, Kirwan CJ, Ackland GL, Pearse RM, Prowle JR. Acute kidney injury and mortality 1 year after major non-cardiac surgery. Br 7 Surg 2017; 104: 868-876.

22 STARSurg Collaborative. A novel risk score to predict post-operative acute kidney injury in patients undergoing major gastrointestinal surgery. A national prospective observational cohort study and external validation. Br 7 Surg Open 2018; 2: 400-410.

23 Modasi A, Pace D, Godwin M, Smith C, Curtis B. NSAID administration post colorectal surgery increases anastomotic leak rate: systematic review/meta-analysis. Surg Endosc 2019; 33: 879-885.

24 Thiels CA, Anderson SS, Ubl DS, Hanson KT, Bergquist WJ et al. Wide variation and overprescription of opioids after elective surgery. Ann Surg 2017; 266: 564-573.

25 Chapman SJ, Thorpe G, Vallance AE, Harji DP, Lee MJ, Fearnhead NS; Association of Coloproctology of Great Britain and Ireland Gastrointestinal Recovery Group. Systematic review of definitions and outcome measures for return of bowel function after gastrointestinal surgery. B7S Open 2018; 3: 1-10.

26 Klein M, Krarup PM, Burcharth J, Ågren MS, Gögenur I, Jorgensen LN et al. Effect of diclofenac on cyclooxygenase-2 levels and early breaking strength of experimental colonic anastomoses and skin incisions. Eur Surg Res 2011; 46: $26-31$.

\section{Supporting information}

Additional supporting information can be found online in the Supporting Information section at the end of the article. 